\title{
Effects of variable loading conditions on the dynamic behaviour of planetary gear with power recirculation
}

\author{
Ahmed Hammami ${ }^{1,2}$, Alfonso Fernandez Del Rincon ${ }^{2}$, Fakher Chaari ${ }^{1}$, Miguel Iglesias \\ Santamaria $^{2}$, Fernando Viadero Rueda $^{2}$, Mohamed Haddar ${ }^{1}$ \\ ${ }^{1}$ Mechanics, Modeling and Production Laboratory- National School of Engineers Sfax \\ BP 1173 - 3038 - Sfax - Tunisia \\ ${ }^{2}$ Department of Structural and Mechanical Engineering - Faculty of Industrial and \\ Telecommunications Engineering - University of Cantabria - Avda. de los Castros s/n - 39005 \\ Santander- Spain. \\ ahmed.hammami2109@gmail.com,alfonso.fernandez@unican.es, \\ fakher.chaari@gmail.com,miguel.iglesias@unican.es,fernando.viadero@unican.es, \\ mohamed.haddar@enis.rnu.tn
}

\begin{abstract}
Variable loads to which gearboxes are subjected are considered as one of the main sources of nonstationarity in these transmissions. In order to characterise their dynamic behaviour in such conditions, a torsional lumped parameter model of a planetary gear with power recirculation was developed. The model included time varying loading conditions and took into account the non-linearity of contact between teeth. The meshing stiffness functions were modelled using Finite Element Method and Hertzian contact theory in these conditions. Series of numerical simulations was conducted in stationary conditions, with different loading conditions. Equation of motion was solved using Newmark algorithm. Numerical results agreed with experimental results obtained from a planetary gear test bench. This test bench is composed of two similar planetary gears called test planetary gear set and reaction planetary gear set which are mounted back-to-back so that the power recirculates through the transmission. The external load was applied through an arm attached to the free reaction ring. Data Acquisition System acquired signals from accelerometers mounted on the rings and tachometer which measured instantaneous angular velocity of the carrier's shaft. The signal processing was achieved using LMS Test.Lab software. Modulation sidebands were obtained from the ring acceleration measurements as well as a non-linear behaviour in case of variable loading resulted by a transfer of the spectral density from the fundamental mesh stiffness to its second harmonic.
\end{abstract}

Keywords back-to-back planetary gears, variable load, dynamic behaviour 


\section{Introduction}

The use of planetary gears in industrial applications is justified by their ability to transmit high torques with substantial ranges of speed reduction. Many industrial applications use this kind of transmissions such as aircraft engines, wind turbines, ...

Several studies were devoted mostly to planetary gears running under constant speed and load i.e. in stationary conditions [1-5]. However, it is possible to find industrial applications involving planetary gears where both load and speed are time varying. Severe non-stationary conditions may lead to excessive vibrations and instability [6].

McFadden and Smith [1] focused on the modulating sidebands around meshing frequency and harmonics caused by non-stationary conditions. Al-shyyab and Kahraman [2] implemented multi-term harmonic balance methodology on a nonlinear torsional model of a single stage planetary gear in order to solve the equations of motion. Inalpolat [3] developed a numerical model to predict modulation sidebands, he described the mechanisms causing sidebands and he validated experimentally the obtained numerical results. He also studied amplitude and frequency modulations caused by manufacturing errors of gears and estimated dynamic loads on sun-planet and ring-planet gear meshes [4]. Liu et al. [5] took into account in their dynamic model variable transmission path of vibration in planetary gears and validated their work experimentally.

Concerning studies devoted to variable loading conditions, it seems that Randall [7] was the first to relate load fluctuation to vibration level. Chaari [8] proposed a bi-dimensional model of a spur planetary gear subjected to both time varying load and speed and highlighted corresponding amplitude and frequency modulations. Mark [9] was interested in the variability of load transmission from one planet to the other caused by imperfections in the transmission. He also focused on vibration spectra shapes of signals registered by fixed-accelerometer for the case of a cracked planet-carrier plate that caused variability in load transfer through planets. Mark [10] extended his studies to the influence of planet-carrier vibration signals modulations on the computed spectra. Feng [11] considered, in his dynamic model of planetary gear, the effect of distributed and localized gear faults on amplitude and frequency modulation effects observed in vibration signals. Lei [12] implemented the adaptive stochastic resonance method for a planetary gearbox having a chipped and missing sun gear tooth. Bartelmus and Zimroz [13] showed that planetary gearbox running in bad conditions is more susceptible to external load than planetary gearbox running in good conditions. They introduced a new diagnostic feature which is used for monitoring the condition of planetary gearboxes under varying external load conditions [14]. Kim [15] was interested in the influence of time varying pressure angles and contact ratios on the dynamic behaviour of planetary gear. In all these cited woks, the varying external load caused a variation of speed in the studied systems.

Complex planetary gearboxes received great interest by scientists. Ligata et al. [16] studied on a back-to-back planetary gear system the influence of manufacturing defects on stresses in the tooth roots and on the load sharing between planets. Singh et al. [17] focused on the impact of changing 
parameters of multistage planetary gearbox on stresses in gear teeth and on load sharing through numerical and experimental investigations. Hammami et al. [18] was interested in the modal characteristics of back-to-back planetary gear set by computing modal kinetic and strain energy distributions. In addition, they [19] achieved a series of experimental tests for run up and run down regimes of the same gearbox in order to validate the modal analysis and to study its dynamic behaviour in non-stationary conditions.

This paper is dedicated to the study of the dynamic behaviour of a complex planetary gearbox running in another non-stationary operation which is the time varying loading condition with the specifications of an imposed constant speed. To achieve this target, a back-to-back planetary gearbox with mechanical power recirculation set up was characterized as the load was applied by the external arm. First, a dynamic model of planetary gear set with power recirculation will be developed. Modulation sidebands will be highlighted in stationary condition in the case of equally-spaced planets and sequentially phased gear meshes. Then, different loading conditions will be considered to show the non linear behaviour of the studied gearbox and to explain its behaviour in the variable loading conditions which is presented in the last section. Simulation and experimental studies for this gear system will be presented and correlated in all studied conditions.

\section{Material and methods}

The test bench used in this research work is composed of two similar planetary gears mounted back-to-back so that the power recirculates through the transmission. This special configuration is selected in order to minimize costs and improve energy efficiency. Figure 1 shows a general scheme of the studied transmission.

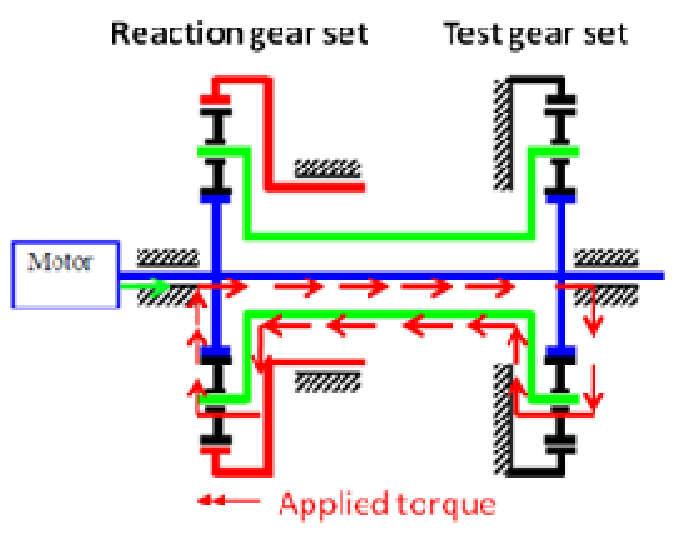

Fig.1: Test bench scheme

The two planetary gear sets are named respectively test gear set and reaction gear set. The main planetary gear set is the test gear set where its output power from its carrier is reintroduced to its sun 
through the reaction planetary gear set. They are connected in back-to-back configuration. Sun gears are mounted on the same shaft and the carriers are connected by a hollow shaft.

In order to introduce external load, mass is added on an arm attached to reaction gear. The test gear ring is clamped (Fig.3). The direction of rotation is such that the friction torque always adds to the reaction ring gear applied torque.

The transmission is driven by an asynchronous motor to which a speed controller is added in order to impose desired speed evolution and values. Accelerations on rings are measured by two tri-axial accelerometers (Fig.2 and Fig.3).

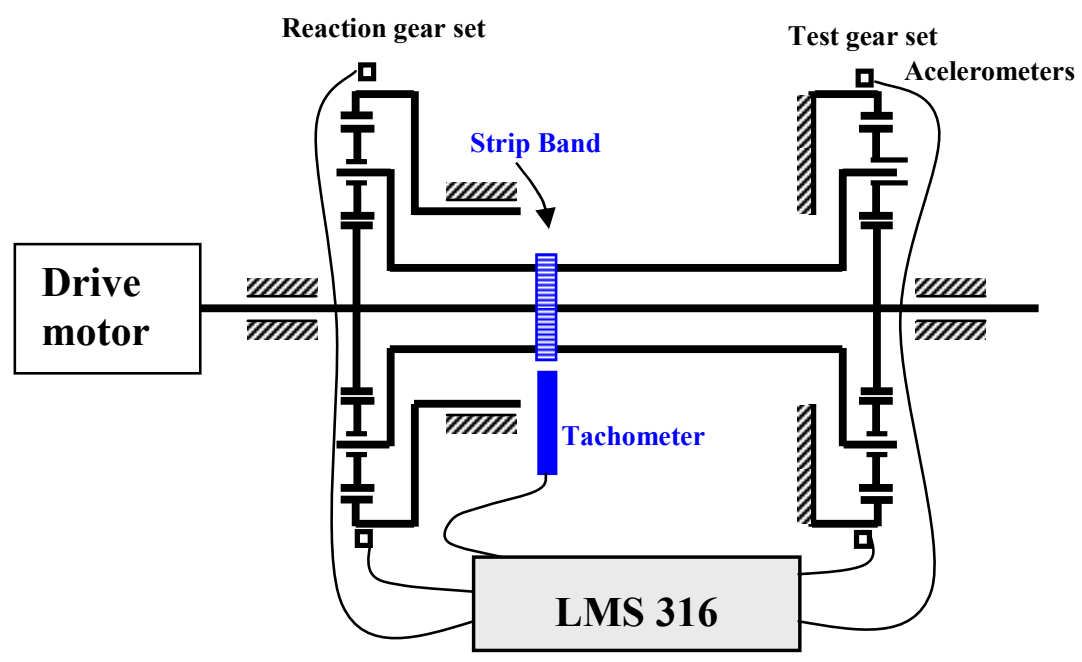

Fig.2: Instrumentation of the test bench

An optical tachometer combined with pulse tapes is mounted on the hollow shaft in order to measure instantaneous angular velocity.

LMS SCADAS 316 Data Acquisition System acquires signals from tachometer and accelerometers and the signal processing is achieved using LMS Test.Lab software. 


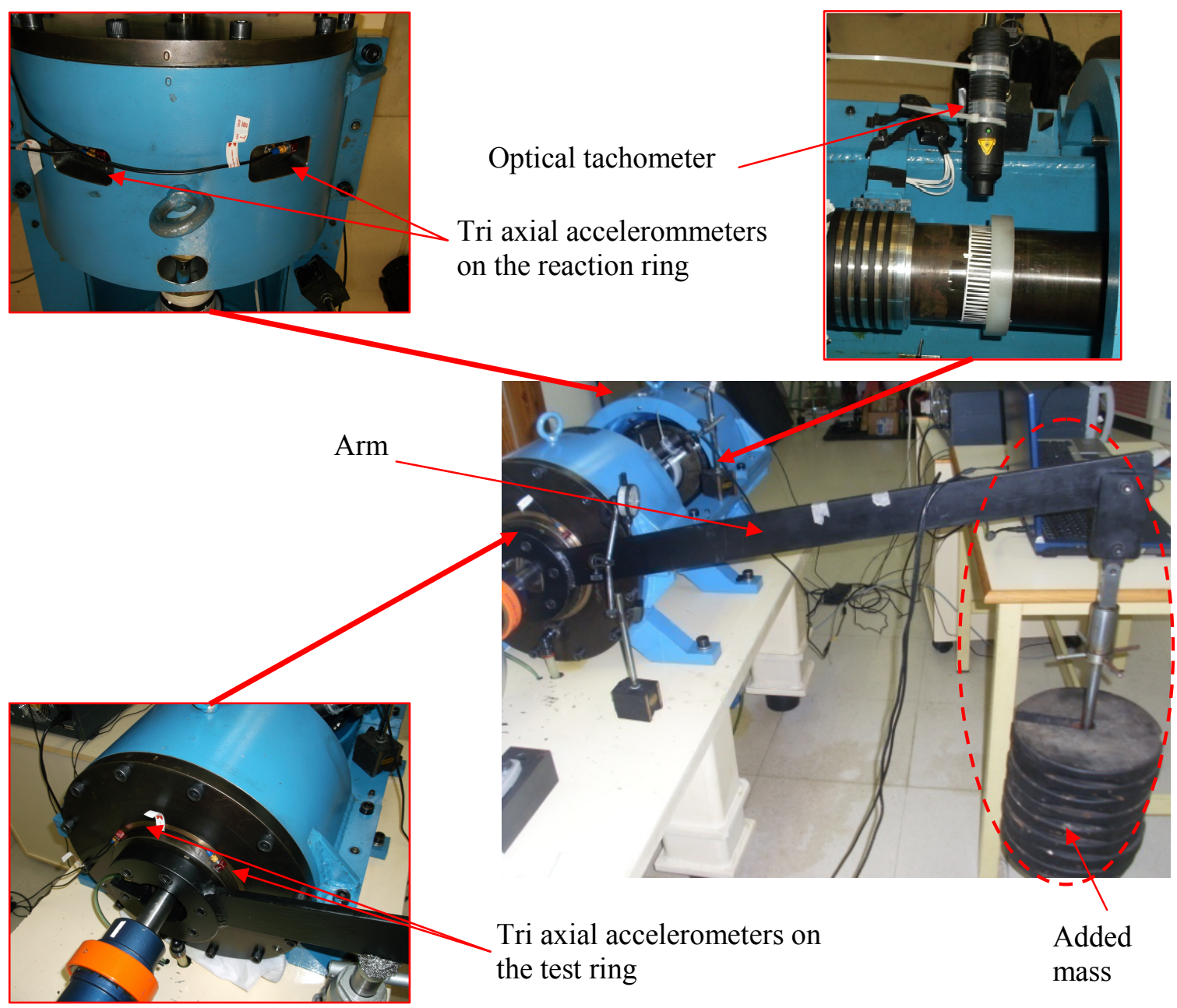

Fig.3: Sensors and external load

International standard "ISO 6336" [20-23] allows to determine the minimum tangential torque on the reaction ring that will produce the mechanical failure in case of tooth bending and pitting for the different gear components of the system. These limits are presented in table 1 .

Table 1. Minimum torque that produce failure

\begin{tabular}{cccc}
\hline Contact & Component & $\begin{array}{c}\text { Torque producing } \\
\text { Bending (N.m) }\end{array}$ & $\begin{array}{c}\text { Torque producing Pitting } \\
\text { (N.m) }\end{array}$ \\
\hline \multirow{2}{*}{ Sun-Planet } & Sun & 2644 & 1100 \\
& Planet & 3630 & 1100 \\
\hline \multirow{2}{*}{ Ring-Planet } & Planet & 2411 & 1650 \\
& Ring & 47587 & 9446 \\
\hline
\end{tabular}

In order to avoid bending and pitting defect, applied torques on the test rig will not exceed $1100 \mathrm{Nm}$. 


\section{Numerical model}

In this section, a dynamic model of the back-to-back planetary gear test rig presented in section 2 will be developed. It is a torsional model based on previous work of Lin and Parker [24]. Figure 4 shows the main components of this model.

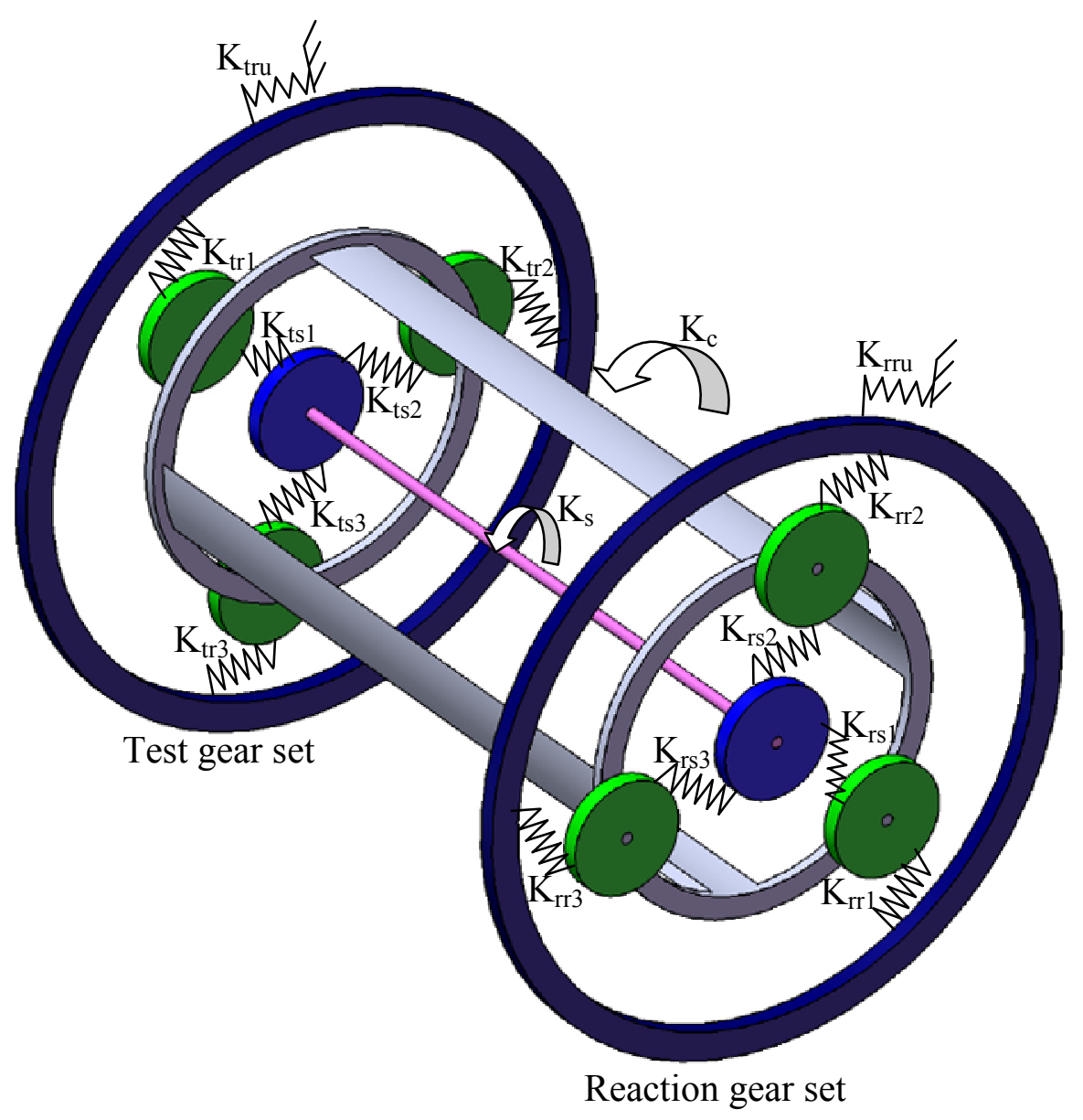

Fig.4: Dynamic model of planetary gear with power recirculation

Mesh stiffness for test ring-planet and test sun-planet are represented respectively by the linear springs $K_{t r, i}$ and $K_{t s, i}$ where $i=1 . . n$ and $n$ is the number of planets ( $\left.n=3\right)$. For the reaction gear set, mesh stiffness is represented by the linear springs $\mathrm{K}_{\mathrm{rr}, \mathrm{i}}$ and $\mathrm{K}_{\mathrm{rs}, \mathrm{i}}$ which are related to reaction ring-planet and reaction-sun planet. Since the reaction ring is free, its torsional stiffness $\mathrm{K}_{\mathrm{rru}}$ is zero, however, the test ring is clamped and modelled by a torsional stiffness $\mathrm{K}_{\text {tru }}$ with high value. The connecting shafts are modelled by torsional stiffness $\mathrm{K}_{\mathrm{s}}$ and $\mathrm{K}_{\mathrm{c}}$. Table 2 shows the parameters of the model. 
Table 2. Parameters of the system

\begin{tabular}{|c|c|c|c|c|}
\hline Planetary gears & Sun & Planet & Ring & Carrier \\
\hline Teeth number & 16 & 24 & 65 & - \\
\hline Mass (Kg) & 0.48 & 1.2 & 28 & 3.6 \\
\hline Base diameter (mm) & 61.3 & 92.0 & 249.3 & 57.5 \\
\hline Moment of inertia $\left(\mathrm{Kg} . \mathrm{m}^{2}\right)$ & $350 \times 10^{-6}$ & $2050 \times 10^{-6}$ & $697770 \times 10^{-6}$ & $21500 \times 10^{-6}$ \\
\hline $\begin{array}{l}\text { Torsional stiffness } \mathrm{Nm} / \mathrm{rd} \\
\text { (test set) } \\
\text { Torsional stiffness }(\mathrm{Nm} / \mathrm{rd}) \\
\text { (Reaction set) }\end{array}$ & & & $\begin{array}{l}3 \times \mathbf{1 0}^{\mathbf{8}} \\
0\end{array}$ & \\
\hline Shaft stiffness & Sun & Planet & Ring & Carrier \\
\hline Torsional (Nm/rad) & $9.3 \times 10^{7}$ & - & - & $3.7 \times 10^{8}$ \\
\hline
\end{tabular}

Non floating Suns and planets are considered and only rotational motions of the gear bodies are considered [25]. The equation of motion of the system with 3 planets can be written as:

$$
M \ddot{X}+C \dot{X}+\left(K(t)+K_{c}\right) X=F(t)
$$

Where $M$ is the mass matrix. $K(t)$ is the stiffness matrix, $K_{c}$ is the coupling shaft stiffness matrix and $F(t)$ is the external force vector applied to the system.

It is possible to decompose $K(t)$ into a mean stiffness matrix $\widetilde{K}$ and a time varying matrix $k(t)$ :

$K(t)=\widetilde{K}+k(t)$

$C$ is the Rayleigh damping matrix expressed by [26]:

$C=\alpha M+\beta \widetilde{K}$

Where $\alpha$ and $\beta$ are two constants [26] expressed by $\alpha=5.10^{-5} s$ and $\beta=5.10^{-4.5} s^{-1}$.

All the matrices are defined in the appendix of [19].

$X$ is the degree of freedom vector expressed by :

$X=\left\{x_{r c}, x_{r r}, x_{r s}, x_{r 1}, x_{r 2}, x_{r 3}, x_{t c}, x_{t r}, x_{t s}, x_{t 1}, x_{t 2}, x_{t 3}\right\}^{T}$

The rotational coordinates are computed by $x_{j r}=r_{j r} \theta_{j r}$ for reaction gear set and by $x_{j t}=r_{j t} \theta_{j t}$ for test gear set $(\mathrm{j}=\mathrm{c}, \mathrm{r}, \mathrm{s}, 1,2,3) . \theta_{j r}$ and $\theta_{j t}$ are the rotational components; $r_{j r}$ and $r_{j t}$ are the base radius for the sun, ring and planets and the radius of the circle passing through the planets centres for the carrier. In order to compute the solution of the problem, implicit Newmark algorithm is used. 


\section{Results and discussion}

Two kinds of simulations and experimental studies will be carried out. In the first one, stationary condition will be considered. Then, time varying loading conditions is applied to the system. Results from simulations and experiments will be compared.

\subsection{Stationary conditions}

Three equally-spaced planets are considered for each planetary gear. They are positioned at angles $\psi_{j n}(\mathrm{n}=1,2,3$ and $\mathrm{j}=\mathrm{r}, \mathrm{t})$. The positions of the 3 planets of the reaction gear set measured with respect to the rotating frame linked to the carrier are $(0,2 \pi / 3,4 \pi / 3)$ whereas the position of the 3 planets of test gear set are $(\pi / 3, \pi, 5 \pi / 3)$. Sequentially phased gear meshes are considered for the two sets since $\frac{Z_{r} \psi_{j n}}{2 \pi} \neq k$ and $\sum_{n=1}^{N} Z_{r} \psi_{j n}=m \pi$ where $\mathrm{k}$ and $\mathrm{m}$ are integer.

Finite elements modelling and Hertzian contact theory are used to define the meshing stiffness functions for all sun-planet and ring-planet meshes following the procedure given by Fernandez et al. [27].

First of all, the tooth geometry is defined using gear parameters which are the gear module, the pressure angle, the addendum, the dedendum and the radius of the rounding tip.

Then, in order to locate the contact points, separation distance between these points is computed in the line of action direction. Considering the analytic formulation of the involuteinvolute contact, the location of the contact points was performed and the deflection of contact points is computed. To generate involute-involute teeth profiles, Litvin theory is considered [28].

Two phenomena are considered: the nonlinear local deformations near the contact area and the tooth body deflections due to linear bending, shearing and compression. The approach used in this work combines an analytical non-linear formulation of Hertzian type for local deflections and a two-dimensional finite element model in order to obtain the global ones.

Finally, the meshing forces are computed. Once the initial separations of the contact points are a function of the position of each gear, the gear meshing forces is obtained imposing the compatibility condition of the displacements.

The procedure adopted to obtain the meshing forces allows the evaluation of the meshing forces, the transmission error and mesh stiffness [27].

Fig.5 and Fig.6 show respectively time varying gear mesh stiffness sun-planet and ring-planet. 


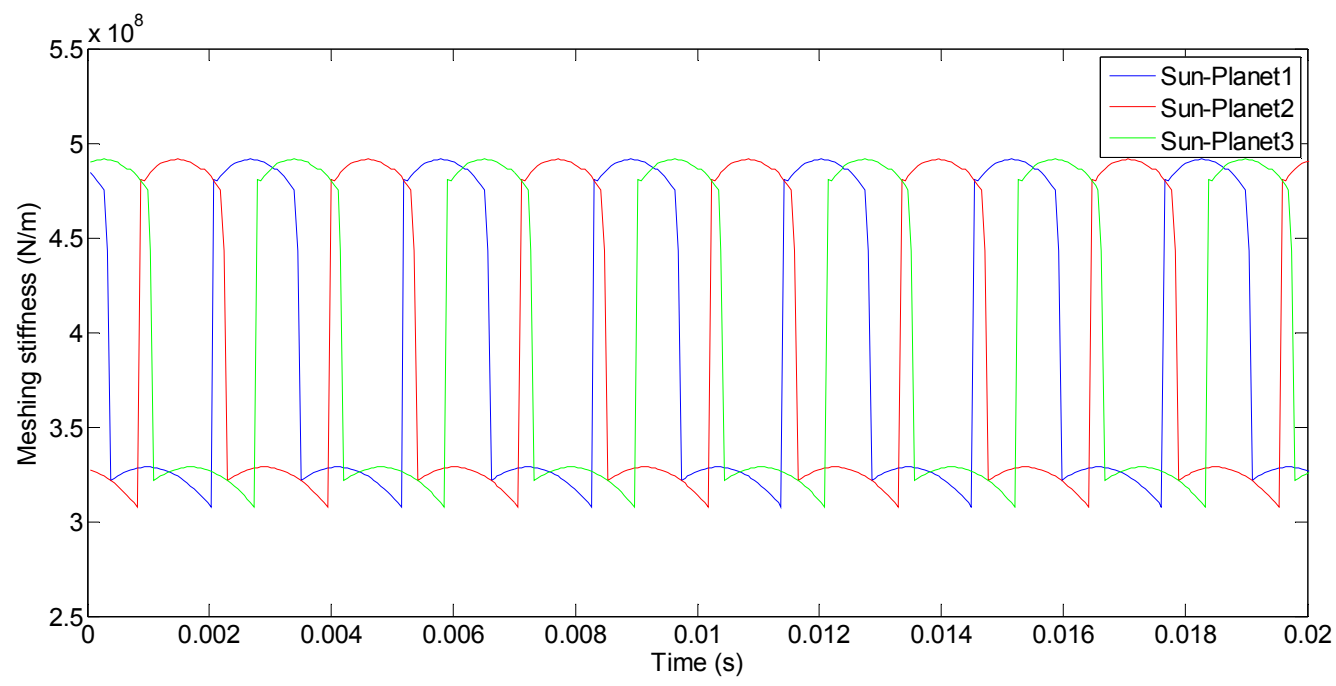

Fig.5: Sun-planet gear mesh stiffness functions in the test gear set

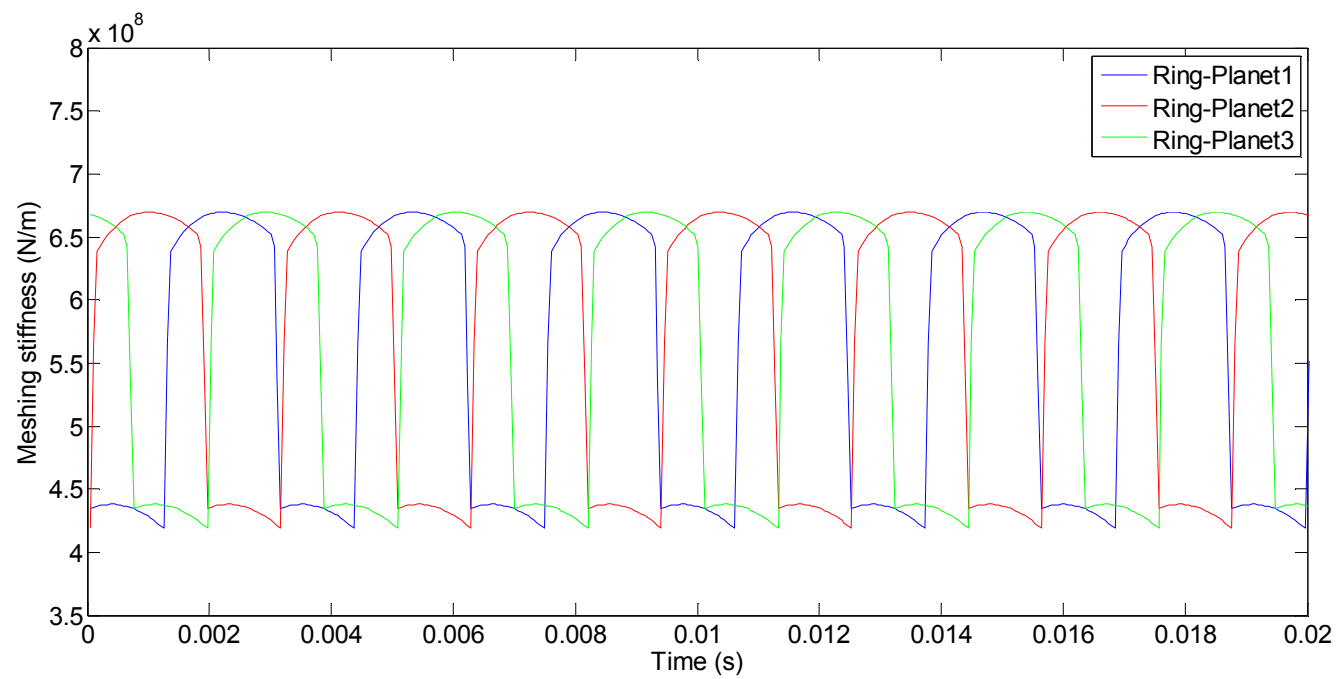

Fig.6: Ring-planet gear mesh stiffness functions in the test gear set

It is clearly noticed the fluctuation of the mesh stiffness values from maximum values corresponding to two teeth pairs in contact to minimum values corresponding to one teeth pair in contact.

The periodicity of the passage of each planet close to the accelerometer position is assumed to be $\mathrm{T}_{\mathrm{c}} / \mathrm{N}(\mathrm{N}=3$ : number of planets). So, if a planet $\mathrm{i}$ approaches the location of the accelerometer placed on the outer part of the ring, its influence on the measured vibration will increase and reach maximum when the planet is close to the sensor and decrease when it rotates far from it. The same phenomenon will be repeated for next planets [3].

" $F_{r}(t)$ " and " $F_{t}(t)$ " are respectively forces induced by the rotation of carriers on the reaction ring and the test ring gear. They are counter-phased. In fact, if a test planet is close to accelerometer mounted on the test ring, the vibration level will be higher; however it will be close to zero for the reaction gear since there is no reaction planet close to accelerometer mounted on the reaction ring. 
This fact is induced by the $\frac{\pi}{3}$ phasing between planets of test and reaction gear sets.

The external force vector is defined by:

$F(t)=\left[\frac{T_{r c}}{r_{r c}}, \frac{T_{r r}}{r_{r r}}+F_{r}(t), \frac{T_{r s}}{r_{r s}}, 0,0,0, \frac{T_{t c}}{r_{t c}}, \frac{T_{t r}}{r_{t r}}+F_{t}(t), \frac{T_{t s}}{r_{t s}}, 0,0,0\right]$

Where $\mathrm{T}_{\mathrm{rc}}, \mathrm{T}_{\mathrm{rr}}, \mathrm{T}_{\mathrm{rs}}, \mathrm{T}_{\mathrm{tc}}, \mathrm{T}_{\mathrm{tr}}$ and $\mathrm{T}_{\mathrm{ts}}$ are respectively the applied torque on the reaction carrier, reaction ring, reaction sun, test carrier, test ring and test sun.

\subsubsection{Constant loaded planetary gear}

In this first test, a simulation is achieved for a fixed speed (1500 rpm) and fixed external load (100 N.m on the free reaction ring). These parameters are also used for the test rig. Figure 7 shows the evolution of acceleration on the fixed ring obtained by simulation and experimental measurements for a unique period of carrier rotation $\left(\mathrm{T}_{\mathrm{c}}=0.2027 \mathrm{~s}\right)$.

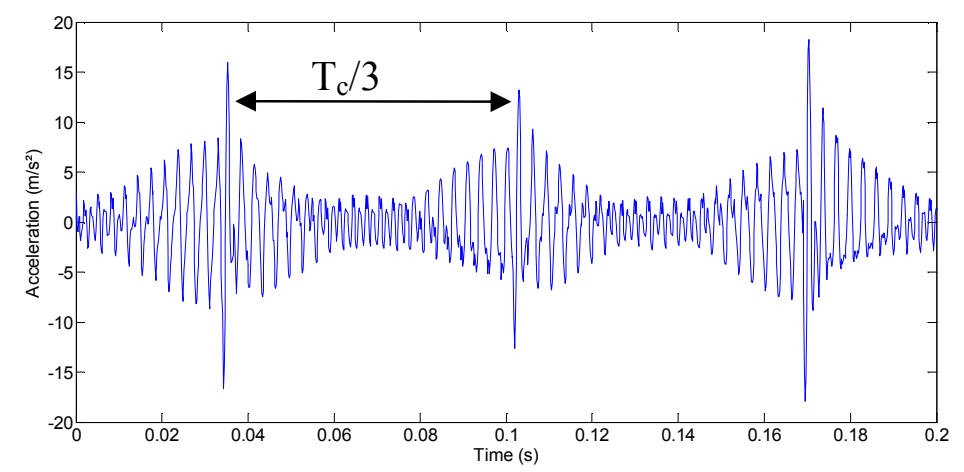

(a)

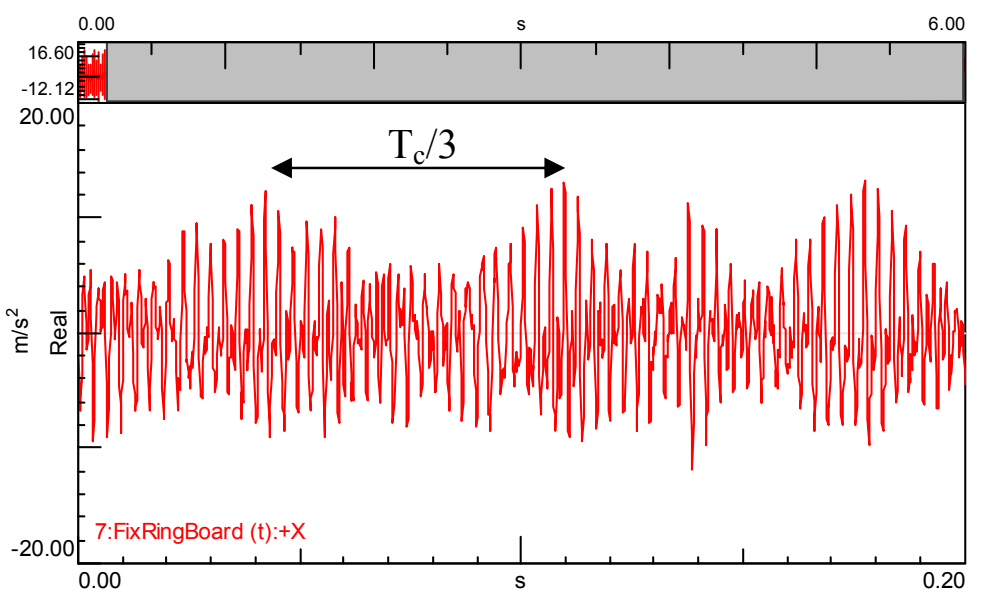

(b)

Fig.7: Acceleration of fixed ring

(a) simulation (b) experimental

Figure 7 shows a clear amplitude modulation [3] which can be explained by the fact that force induced by the rotation of carrier at $\mathrm{T}_{\mathrm{c}}=0.2027 \mathrm{~s}$ and $\mathrm{f}_{\mathrm{c}}=4.93 \mathrm{~Hz}$ modulates the mesh signal.

The corresponding spectra (Fig.8) show several peaks (sidebands) close to the mesh frequency $(320.66 \mathrm{~Hz})$ and its harmonics which are located at 3.n. $\mathrm{f}_{\mathrm{c}}$ and $\mathrm{m} . \mathrm{f}_{\mathrm{c}}(\mathrm{n}$ and $\mathrm{m}$ integers). 

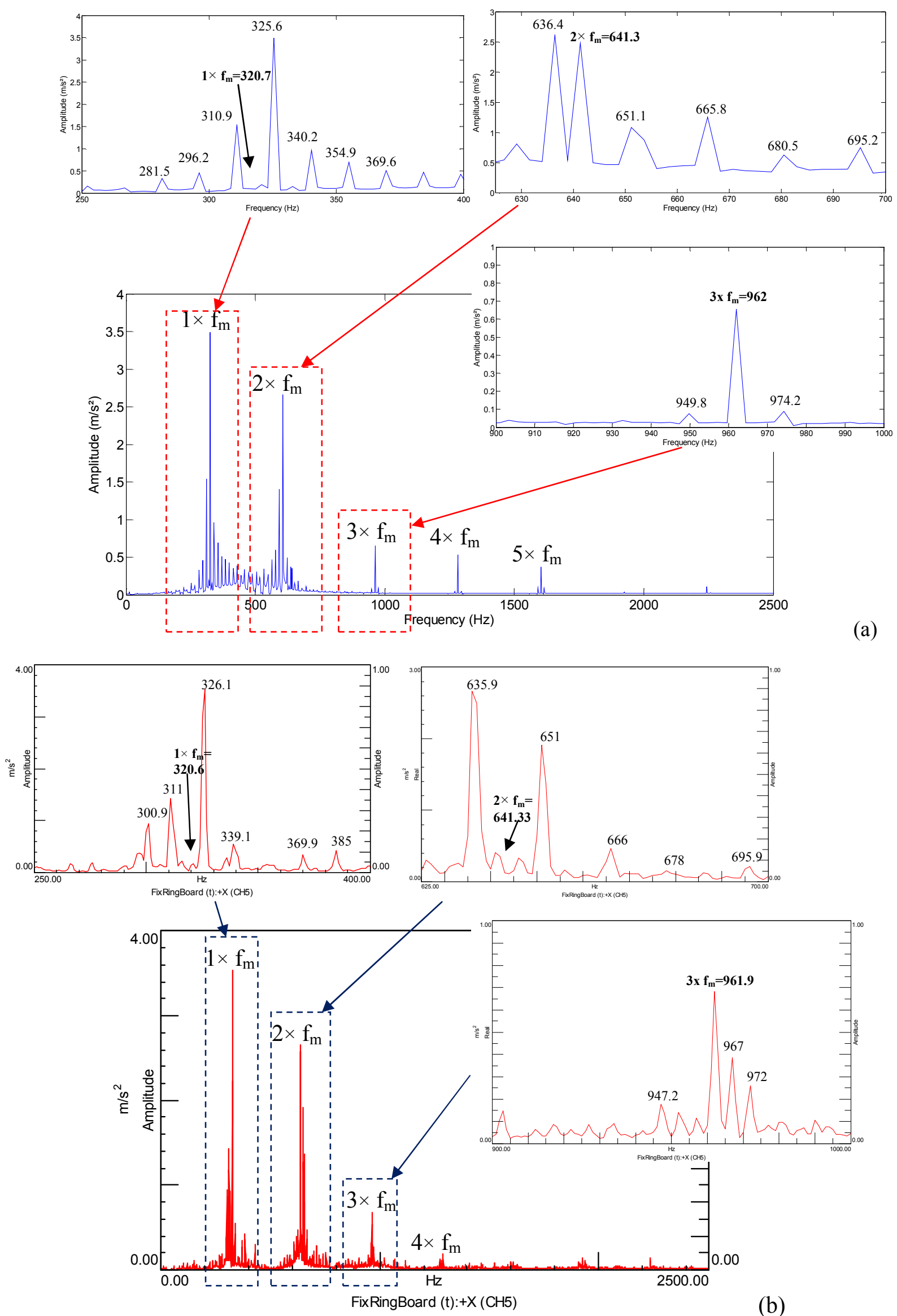

(b)

Fig.8: Spectra of acceleration on the fixed ring: Theoretical (a) and experimental (b) 
Sidebands are not symmetric around $1 \mathrm{xf}_{\mathrm{m}}$. The highest amplitudes are identified at frequencies $\mathrm{f}=\mathrm{n} . \mathrm{N} . \mathrm{f}_{\mathrm{c}}=\mathrm{n} \times 3 \times 4.9=\mathrm{n} \times 14.7 \mathrm{~Hz}$ ( $n$ : integer) which corresponds to $325.6 \mathrm{~Hz}$. This result confirms what was explained by McFadden and Smith in [1].

For $2 \mathrm{x}_{\mathrm{m}}$ the highest sideband amplitude is on the left, however, for $3 \times \mathrm{f}_{\mathrm{m}}$ the maximum amplitude is right on this mesh frequency harmonic as shown in the different zooms of Fig. 8.

\subsubsection{Different loading conditions of the planetary gear}

In this section, different loading conditions are considered: 100N.m, 300N.m, 500N.m, 700N.m and 900 N.m. Finite elements method is used to define the mesh stiffness functions for each load case [27]. Fig.9 and Fig.10 show examples of mesh stiffness functions corresponding to sun-planet1 and ring-planet.

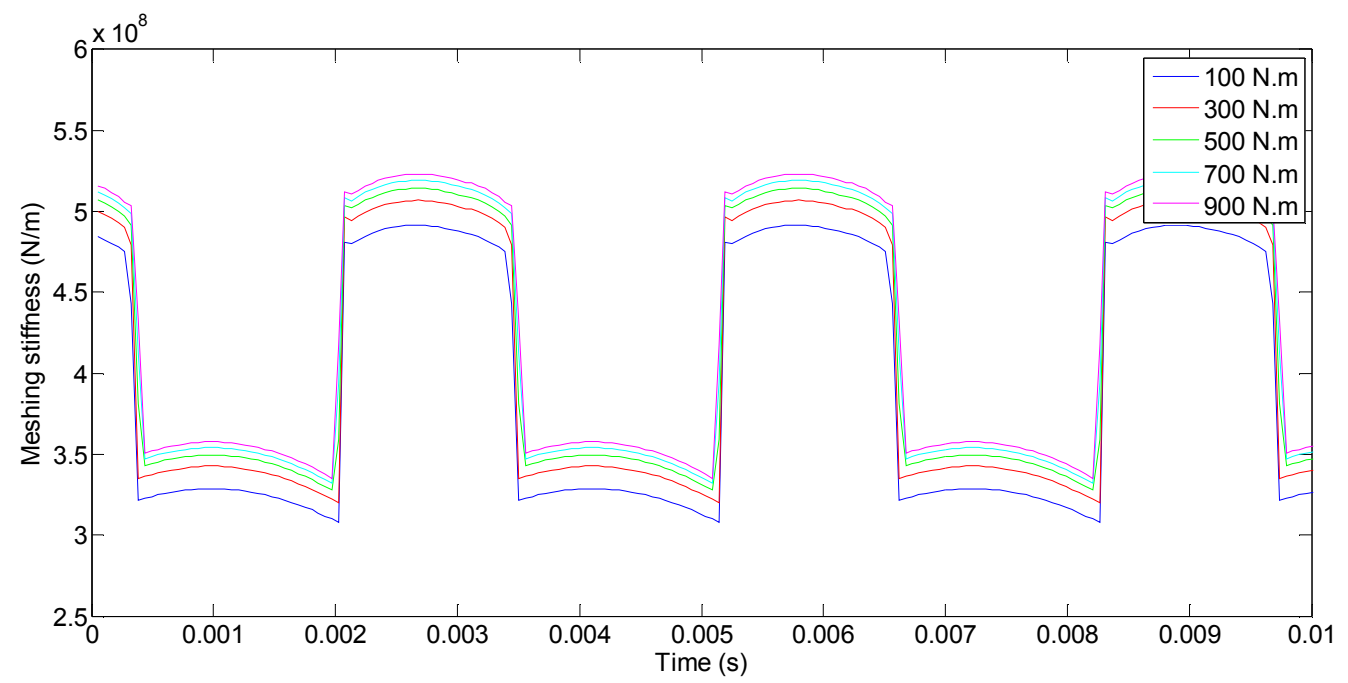

Fig.9: sun-planet1 mesh stiffness functions for test gear set

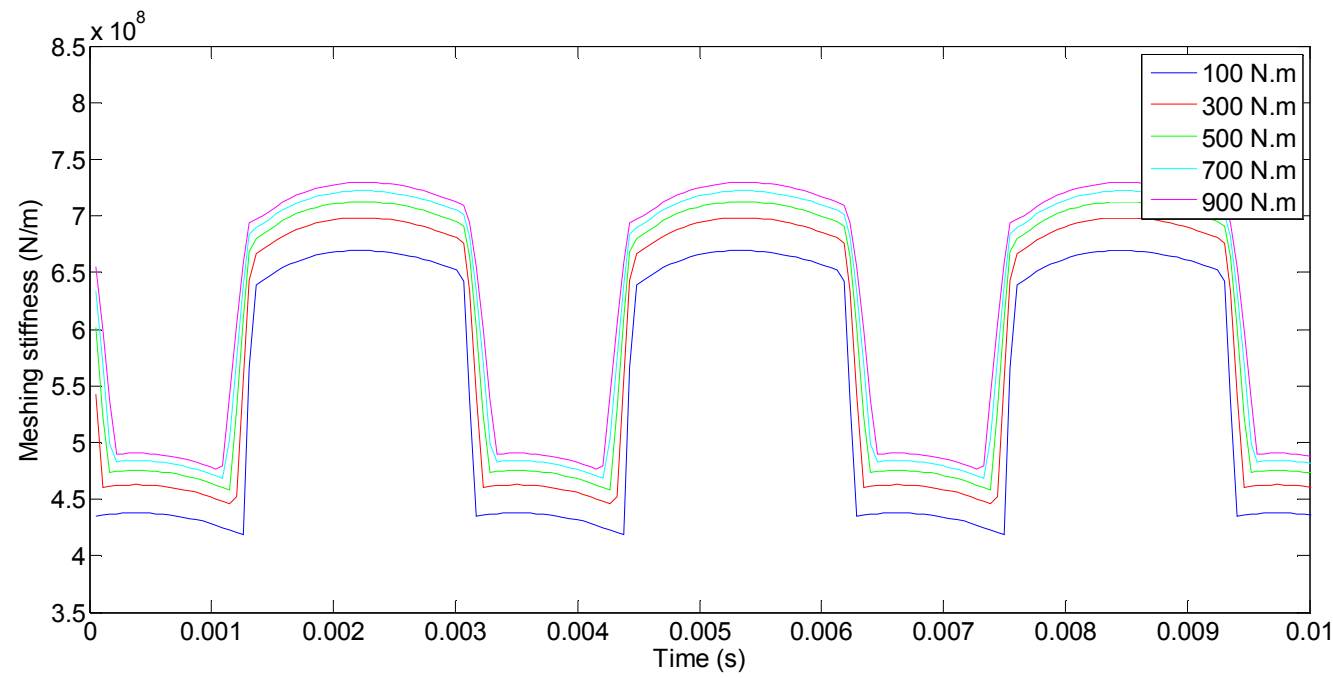

Fig.10: Ring-planet1 mesh stiffness functions for test gear set 
In Fig. 9 and Fig.10, the period relative to double teeth contact increases as the load level increases, this leads also to an increase in the contact ratio. It is also possible to observe the effect of the tip rounding in the transition zone, where a sharp increase on the meshing stiffness is found. That effect is a consequence of a singularity in the local deformation as the curvature radius of the profile changes from a small value for the tip rounding to a higher one corresponding to the involute profile. So, the nonlinearity induced by local Hertzian contact model has strong effect on mesh stiffness function highlighted by the shift of the stiffness curves if applied torque is changed.

Fig. 11 shows that as the load increases, the spectral density moves from $1 \times f_{m}$ to $2 \times f_{m}$.
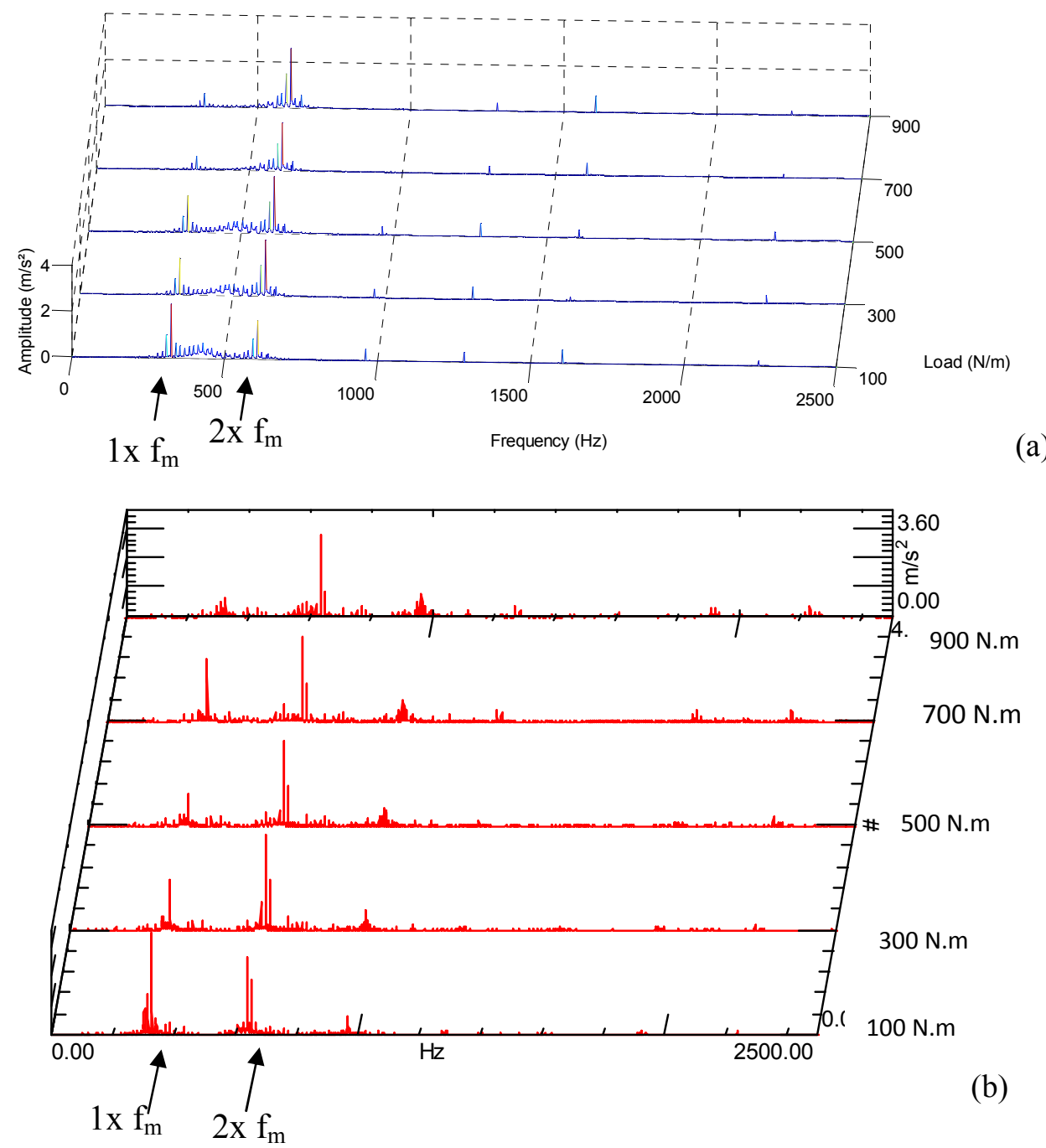

Fig.11: Spectra of acceleration on fixed ring for different loads (a) simulation (b) experimental

In fact, when the torque is increased, meshing forces values increase for each of the contacting points through a meshing period. This causes variation of the pressure angle and therefore an increase of the contact ratio (Fig.9 and Fig.10). So, the period of double contact increases as the torque level increases. Also, the first coefficient of the Fourier series of this input (at the mesh frequency) 
decreases whilst the second coefficient increases. In addition, the inertia of the reaction ring gear increases when mass is added and vice-versa.

\subsection{Variable load}

A time varying loading condition is applied to the free ring as shown in figure 12 .

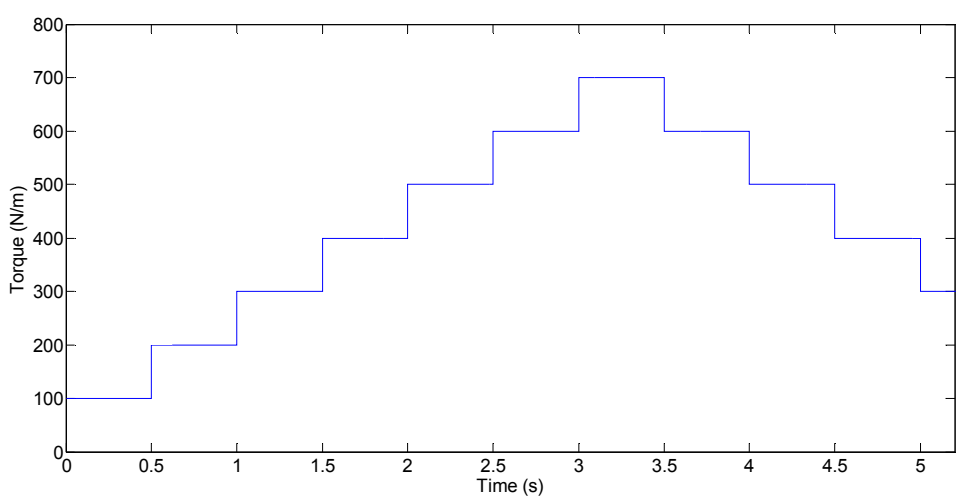

Fig.12: The external variable torque

The variation of the external load implies a variation of the value of the meshing stiffness and a variation of the period of double teeth on contact (Fig.13).

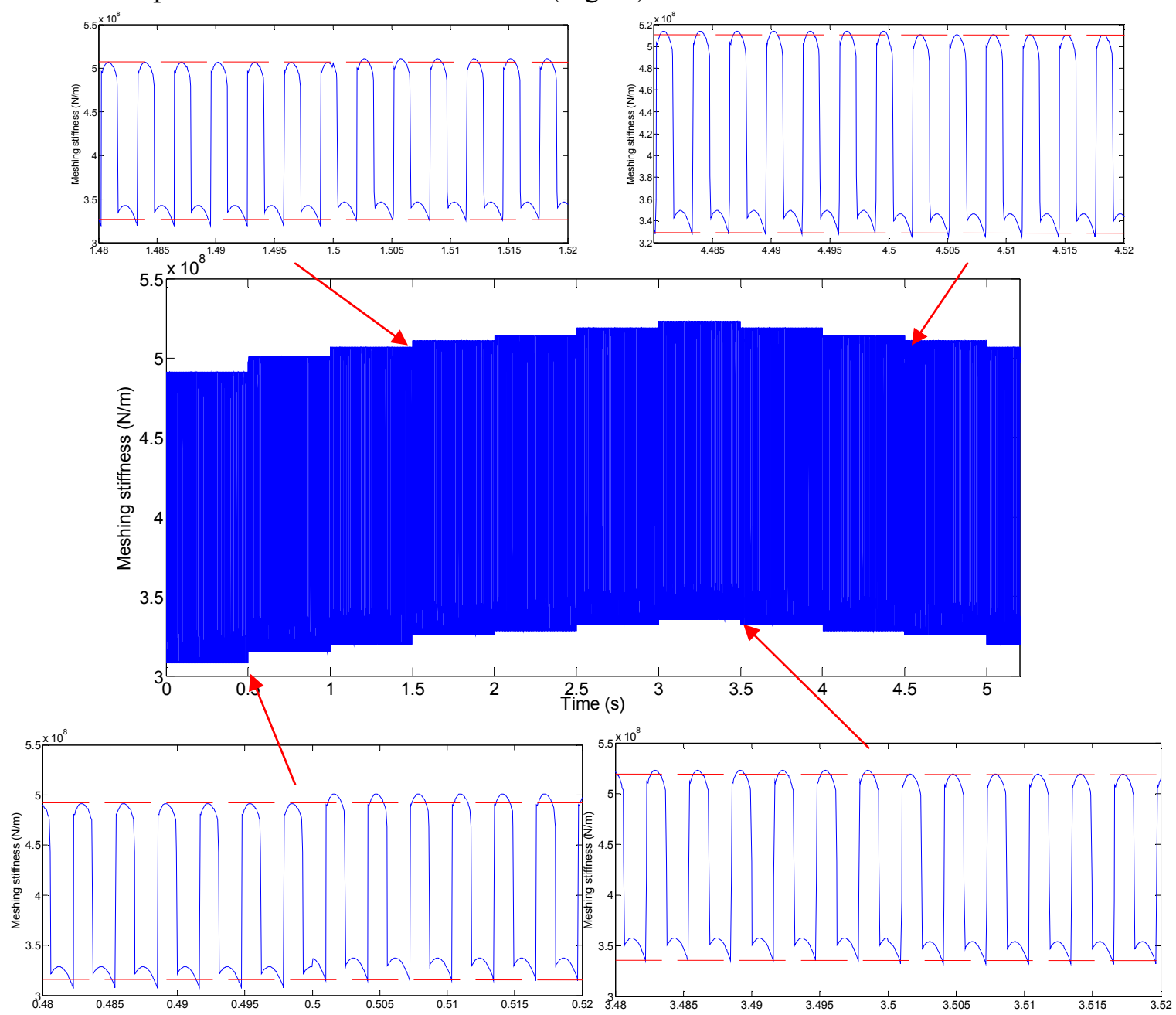

Fig.13: Sun-planet1 mesh stiffness function for the test gear set evolution with variable load 
The speed of motor remains constant as it is controlled by the frequency converter.

The spectrum of acceleration on the fixed ring plotted on figure 14 shows that the nearest harmonic frequency of rotation of carrier to the frequency $2 . \mathrm{f}_{\mathrm{m}}$ is the dominant and corresponds to an intermediate frequency.

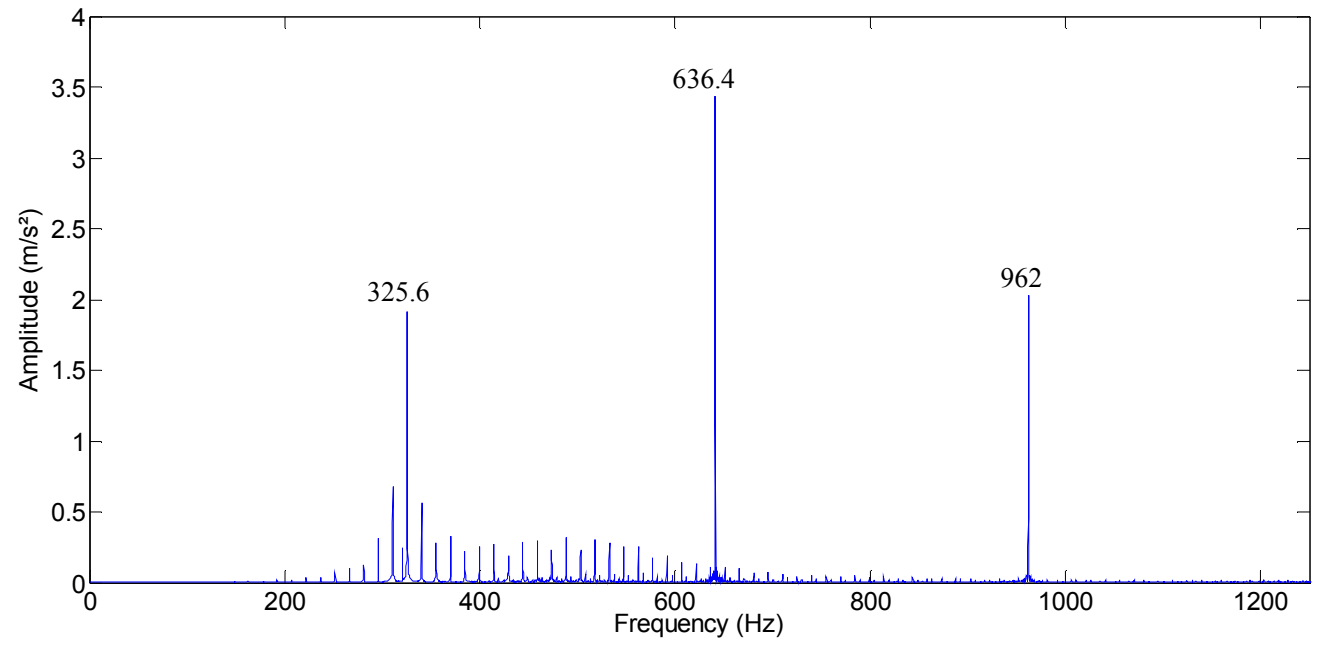

(a)

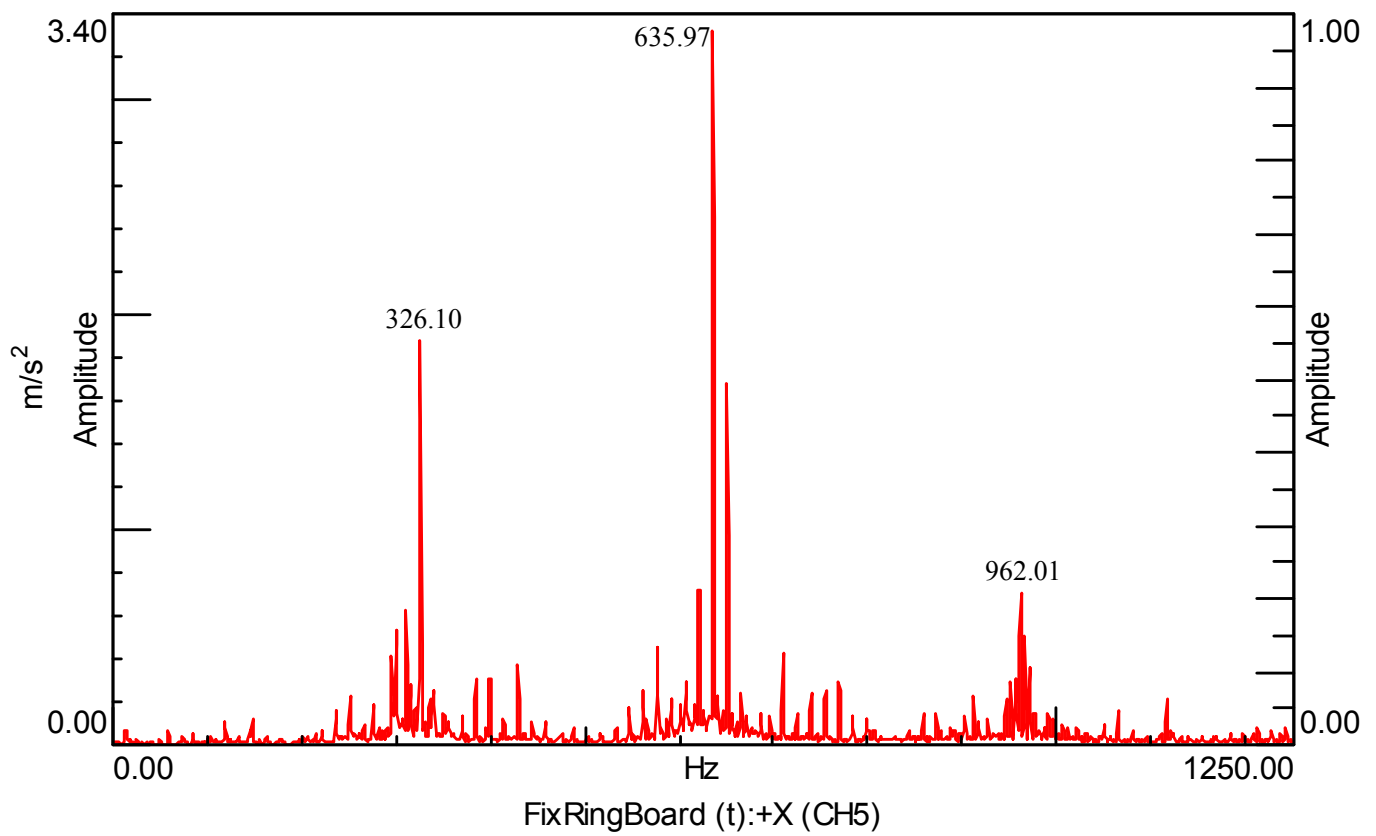

(b)

Fig.14: Response spectrum of the fixed ring with variable load:

(a) simulation (b) experimental

Although the system starts with $100 \mathrm{~N} . \mathrm{m}$ as an external torque where the fundamental meshing frequency is the dominant (Fig.8), the spectrum in figure 14 is likely the same as the spectrum of 500 N.m load which is predicted in Figure 11. In this is case, the variable loading condition can be averaged at this time scale. 


\section{Conclusion}

A back-to-back planetary gear system running under stationary and non-stationary operating conditions was investigated. Both numerical and experimental tests were achieved and an agreement between simulations and the tests was obtained. The test rig has a back-to-back configuration for power recirculation and it allows the modification of the loading torque during test with the aim to reproduce non-stationary conditions. A torsional lumped parameter model of the test bench was developed.

In a first study, stationary operating conditions were considered. The passage's effects of each planet close to the accelerometer position mounted on the ring were taken into account and the mesh stiffness was modelled through the finite element method and the contact hertzian formulation. The dynamic behaviour of planetary gear set in the case of equally-spaced planets and sequentially phased gear meshes was approved; an amplitude modulation was observed in the acceleration time evolution of the test ring and modulation sidebands of the vibration spectra were highlighted by simulation and experiments.

Then, different loading conditions were considered. By adding masses to the free ring (load variation), the inertia of the reaction ring gear changes. Using the finite elements method and the contact hertzian formulation of involute-involute teeth profiles, it was shown that the amplitude of meshing forces and the period of double contact increase as the torque level increases. This was observed in the time evolution of sun-planet and planet-ring mesh stiffness. A transfer of the spectral density from the fundamental mesh stiffness to its second harmonic was observed in the waterfall plot of the different loading accelerations on the test ring and was explained by the fact that the first coefficient of the Fourier series of the mesh stiffness decreases whilst the second coefficient increases as the torque level increases. This behaviour was the results of an increase the pressure angle and the contact ratio causing an expansion of the period of double contact between teeth.

In case of variable loading conditions, an increase of the external load implies an increase of the amplitude of the meshing stiffness and the period of double teeth on contact and vice-versa. A nonlinearity of the contact between teeth was found in the evolution of the mesh stiffness functions. The dynamic behaviour of back-to-back planetary gear changes due to the change of mass and stiffness with an amplification of the $2^{\text {nd }}$ harmonic of the meshing frequency despite the system was started with a low external torque.

In future investigations, the influence of time range loading conditions on the dynamic behaviour of the system will be focused in the case Involute - Tip rounding contact between teeth. 


\section{Acknowledgements}

This work was financially supported by the Tunisian-Spanish Joint Project No. A1/037038/11.

The authors would like also to acknowledge the project funded by the Spanish Ministry of Science and Technology and called "Development of methodologies for the simulation and improvement of the dynamic behavior of planetary transmissions DPI2013-44860".

\section{References}

[1] P.D. McFadden and J.D. Smith, An explanation for the asymmetry of the modulation sidebands about the tooth meshing frequency in epicyclic gear vibration, Proceedings of the Institution of Mechanical Engineers 199 C1 (1985)65-70.

[2] A. Al-shyyab and A. Kahraman, A non-linear dynamic model for planetary gear sets, Proceedings of the Institution of Mechanical Engineers K: Journal of Multi-body Dynamics, 221 (2007) 567-576

[3] M. Inalpolat, A. Kahraman, A theoretical and experimental investigation of modulation sidebands of planetary gear sets, Journal of Sound and Vibration 323 (2009) 677-696

[4] M. Inalpolat, A. Kahraman, A dynamic model to predict modulation sidebands of a planetary gear set having manufacturing errors, Journal of Sound and Vibration 329 (2010) 371-393

[5] L. Liu, X. Liang, M.J. Zuo, Vibration signal modeling of a planetary gear set with transmission path effect analysis, Measurement 85 (2016) 20-31

[6] Y. Lei, J. Lin, M.J. Zuo, Z. He, Condition monitoring and fault diagnosis of planetary gearboxes: a review, Measurement, 48 (2014) 292-305

[7] R.B. Randall, A new method of modelling gear faults, Journal of Mechanical Design, 104 (1982) 259-267

[8] F. Chaari, M. S. Abbes, F. Viadero Rueda, A. Fernandez del Rincon, M. Haddar, Analysis of planetary gear transmission in non-stationary operations, Frontiers Mechanical Engineering, 8 (2013) 88-94

[9] W.D. Mark, J.A. Hines, Stationary transducer response to planetary-gear vibration excitation with non-uniform planet loading, Mechanical Systems and Signal Processing 23 (2009) 13661381

[10] W.D. Mark, Stationary transducer response to planetary-gear vibration excitation II: Effects of torque modulations, Mechanical Systems and Signal Processing 23 (2009) 2253-2259

[11] Z. Feng , M.J. Zuo, Vibration signal models for fault diagnosis of planetary gearboxes, Mechanical Systems and Signal Processing 36 (2013)401-421

[12] Y. Lei, D. Han, J. Lin, Z. He, Planetary gearbox fault diagnosis using an adaptive stochastic resonance method, Mechanical Systems and Signal Processing (2012), http://dx.doi.org/10.1016/j.ymssp.2012.06.021

[13] W. Bartelmus, R. Zimroz, Vibration condition monitoring of planetary gearbox under varying external load, Mechanical Systems and Signal Processing, 23 (2009) 246-259

[14] W. Bartelmus , R. Zimroz, A new feature for monitoring the condition of gearboxes in nonstationary operating conditions, Mechanical Systems and Signal Processing, 23 (2009) 15281534 
[15] W. Kim, J.Y. Lee, J. Chung, Dynamic analysis for a planetary gear with time-varying pressure angles and contact ratios, Journal of Sound and Vibration 331 (2012) 883-901

[16] H. Ligata, A. Kahraman, A. Singh, An Experimental Study of the Influence of Manufacturing Errors on the Planetary Gear Stresses and Planet Load Sharing, Journal of Mechanical Design 130 (2008) 041701

[17] A. Singh, A. Kahraman, H. Ligata, Internal Gear Strains and Load Sharing in Planetary Transmissions: Model and experiments, Journal of Mechanical Design 130 (2008)072602

[18] A. Hammami, A. Fernandez, F. Viadero, F. Chaari, M. Haddar, Modal analysis of back-to-back planetary gear: experiments and correlation against lumped parameter model, Journal of Theoretical and Applied Mechanics, 2015,53 (1) 125-138

[19] A. Hammami, A. Fernandez, F. Chaari, F. Viadero, M. Haddar, Dynamic behaviour of backto-back planetary gear in run up and run down transient regimes, Journal of Mechanics, 2015, 31 (4) 481-491

[20] ISO 6336-1, Calculation of load capacity of spur and helical gears, Part 1: Basic principles, introduction and general influence factors. International Standard, (2006).

[21] ISO 6336-2, Calculation of load capacity of spur and helical gear, Part 2: Calculation of surface durability (pitting), International Standard, (2006).

[22] ISO 6336-3, Calculation of load capacity of spur and helical gear, Part 3: Calculation of tooth bendind strength, International Standard, (2006).

[23] ISO 6336-6, Calculation of load capacity of spur and helical gear, Part 6: Calculation of service life under variable load, International Standard, (2006).

[24] J. Lin and R.G. Parker, Planetary gear parametric instability caused by mesh stiffness variation, Journal of Sound and Vibration 249 (2002) 129-145

[25] A. Kahraman, Natural modes of planetary gear trains, Journal of Sound and Vibration, 1994, 173 (1) $125-130$

[26] G.Dhatt, G.Touzot, Une présentation de la méthode des éléments finis, Paris (1984) Editions Maloine.

[27] A. Fernandez del Rincon, F. Viadero, M. Iglesias, P. García, A. de-Juan, R. Sancibrian, A model for the study of meshing stiffness in spur gear transmissions, Mechanism and Machine Theory 61 (2013) 30-58

[28] F.L. Litvin, A. Fuentes, Gear geometry and applied theory, 2nd ed. Cambridge UniversityPress, Cambridge, 2004. 